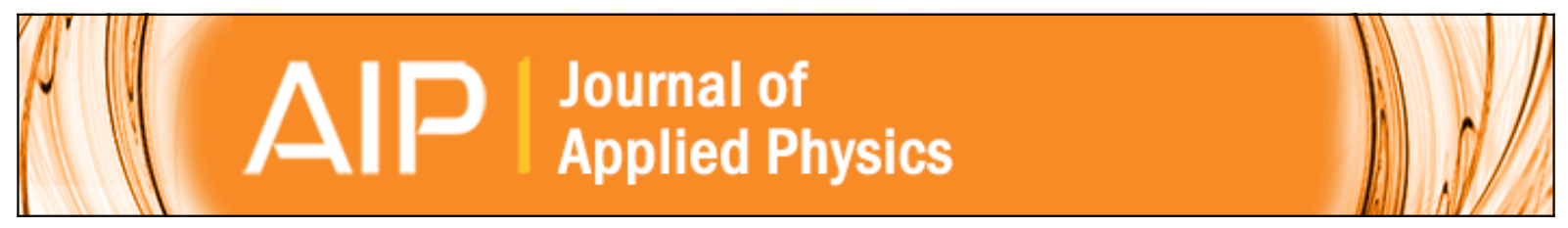

\title{
Flux formulation of hyperbolic heat conduction
}

J. I. Frankel, Brian Vick, and M. N. Özisik

Citation: Journal of Applied Physics 58, 3340 (1985); doi: 10.1063/1.335795

View online: http://dx.doi.org/10.1063/1.335795

View Table of Contents: http://scitation.aip.org/content/aip/journal/jap/58/9?ver=pdfcov

Published by the AIP Publishing

\section{Articles you may be interested in}

Guiding conductive heat flux through thermal metamaterials

Appl. Phys. Lett. 105, 193904 (2014); 10.1063/1.4901885

Large penetration depth of near-field heat flux in hyperbolic media

Appl. Phys. Lett. 104, 121903 (2014); 10.1063/1.4869490

Hyperbolic heat conduction due to axisymmetric continuous or pulsed surface heat sources

J. Appl. Phys. 68, 5478 (1990); 10.1063/1.347006

Hyperbolic heat conduction with temperature-dependent thermal conductivity

J. Appl. Phys. 59, 1861 (1986); 10.1063/1.336413

Variational formulations for heat conduction problems

J. Appl. Phys. 50, 673 (1979); 10.1063/1.326028

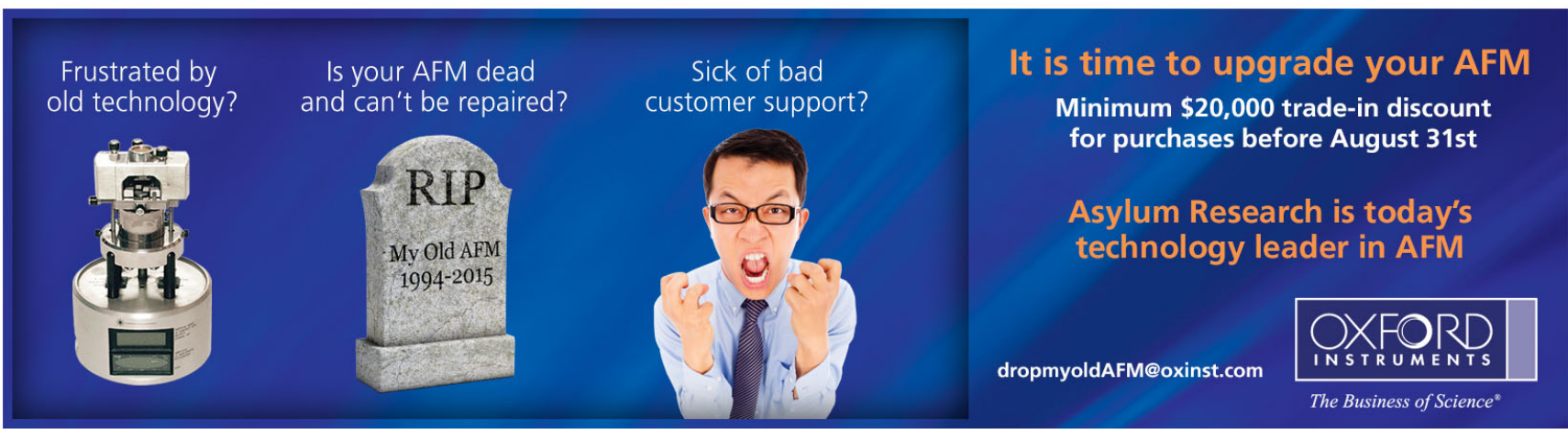




\title{
Fux formulation of hyperbollic hear conduction
}

\author{
J. I. Frankel and Brian Vick \\ Mechanical Engineering Department, Virginia Polytechnic Institute and State University, Blacksburg, \\ Virginia 24061 \\ M. N. Özisik \\ Mechanical and Aerospace Engineering Department, North Carolina State University, Box 7910, Raleigh, \\ North Carolina 27695
}

(Received 8 April 1985; accepted for publication 9 July 1985)

The development of the general flux formulation for heat conduction based on the modified Fourier's law is presented. This new formulation produces a hyperbolic vector equation in heat flux which is more convenient to use for analysis in situations involving specified flux conditions than the standard temperature formulation. The recovery of the temperature distribution is obtained through integration of the energy conservation law with respect to time. The Green's function approach is utilized to develop a general solution for hyperbolic heat conduction in a finite medium. The utility of the fiux formulation and the unusual nature of heat conduction based on the hyperbolic formulation are demonstrated by developing analytical expressions for the heat flux and temperature distributions in a finite slab exposed to a pulsed surface heat flux.

\section{INTRODUCTION}

In classical heat conduction theory, the constitutive equation governing heat flow is given by Fourier's law

$$
q=-k \frac{\partial T}{\partial x}
$$

which states that the heat flux is proportional to the temperature gradient. This law originated from experimental observation. When the constitutive relation, as expressed in Eq. (1), is incorporated into the First Law of Thermodynarnics, a parabolic partial differential equation is obtained. Controversy arises using such a description for linear models in that it predicts that any thermal disturbance on a body is instantaneously felt throughout the body, that is, heat propagates at an infinite speed. Since thermal energy is carried by molecular motion which propagates at a finite speed, one generally concludes that Fourier's law is a low order approximation to a more exact constitutive equation. ${ }^{1-3}$ Despite this apparent paradox, Fourier's law is quite accurate for most common engineering situations. However, for situations involving very short times, extreme thermal gradients, or temperatures near absolute zero, Fourier's law becomes invalid.

It was originally proposed by Maxwell ${ }^{4}$ via kinetic considerations and later by Vernotte, ${ }^{5,6,7}$ Cattanea, ${ }^{8}$ and Morse and Feshbach ${ }^{9}$ on a heuristic basis that the particular law for heat conduction should account for thermal relaxation or the finite buildup time for the onset of heat to flow. The proposed modified Fourier law is

$$
\tau \frac{\partial q}{\partial t}+q=-k \frac{\partial T}{\partial x}
$$

where $\tau$ is the thermal relaxation time, and $k$ is the thermal conductivity.

Since the arguments were originally qualitative, many different approaches have been proposed to quantify Eq. (2). Weymann ${ }^{10}$ and Taitel ${ }^{3}$ obtained Eq. (2) through a random walk argument. Kaliski" modified thermodynamics for an irreversible process. Gurtin and Pipkin ${ }^{12}$ also developed a general theory based on thermodynamics with memory, where an integro-differential equation is derived. Kinetic considerations have been incorporated by Bubnov, ${ }^{13}$ and Berkovsky and Bashtovoi. ${ }^{14}$ Since Fourier's law is inconsistent with special relativity, relativistic models have been proposed by Van Kampen ${ }^{15}$ and Keilly ${ }^{16}$ for heat transport.

Chester ${ }^{2}$ states that Fourier's law is simply an approximation to the modified law. Simons ${ }^{1}$ points out, based on the Boltzmann equation, that a hierarchy of increasing complexity exists in describing the "true" heat conduction equation. He concludes that for the nonrelativistic regime, Eq. (2) has been generally agreed upon as the next approximation.

The nature of heat propagation resulting from Eq. (2) has been studied by many investigators. ${ }^{17-29}$ In all these studies, the temperature distribution was obtained through the temperature field equation. The objective of this exposition is to give an alternative formulation based on heat flux. First, a general three dimensional constant property heat flux formulation with an arbitrary volumetric heat source is developed. Then the utility of such a formulation and the peculiar nature of the hyperbolic theory are illustrated with a specific example involving a finite slab subjected to a pulsed heat flux at one of its surfaces.

\section{I1. GENERAL HEATELUX FORMULATION}

The governing heat flux vector equation is now derived for any orthogonal coordinate system. Performing an energy balance over a control volume, ${ }^{30,31}$ we arrive at

$$
\int_{V}\left(-\nabla \cdot \mathbf{q}(r, t)+u(\bar{r}, t)-\rho \frac{\partial e}{\partial t}(\bar{r}, t)\right) d V=0,
$$

where $u$ represents a volumetric energy source, $e$ is the internal energy, $\rho$ is the density, $t$ is the temporal variable, and $\bar{r}$ is the space vector. For the integral in Eq. (3) to vanish over an arbitrary region, the integrand must be identically zero. Therefore, we arrive at the usual result 


$$
-\nabla \cdot \mathbf{q}(\bar{r}, t)+u(\bar{r}, t)=\rho C_{p} \frac{\partial T}{\partial t}(\bar{r}, t),
$$

which incorporates the assumption that the internal energy is only a function of temperature for an incompressible substance. In general vector notation, the modified Fourier law becomes

$$
\tau \frac{\partial \mathbf{q}}{\partial t}(\bar{r}, t)+\mathbf{q}(r, t)=-\mathbf{k} \nabla T .
$$

Eliminating the temperature $T(\bar{r}, t)$ between Eqs. (4) and (5), with the assumption of constant properties, gives the following heat flux vector equation:

$$
\nabla[\nabla \cdot \mathbf{q}(r, t)]-\nabla u(\bar{r}, t)=\frac{1}{\alpha}\left(\tau \frac{\partial^{2} \mathbf{q}}{\partial t^{2}}(r, t)+\frac{\partial \mathbf{q}}{\partial t}(r, t)\right),
$$

where $\alpha \equiv k / \rho C_{p}$ is the thermal diffusivity. For the threedimensional case, Eq. (6) represents three simultaneous hyperbolic scalar equations for the heat flux components. Note that in this formulation only the gradient of the volumetric heat source is involved in Eq. (6), whereas Vick and Özisik $^{17,18}$ have shown when considering the temperature field formulation that the volumetric source and its time derivative appear in the equation.

Finally, given the heat flux, the temperature distribution is recovered by integrating the general energy balance relation of Eq. (4) over time

$$
\begin{gathered}
T(\bar{r}, t)=T(r, 0)+\int_{t^{\prime}=0}^{t}\left(1 / \rho C_{p}\right)\left[-\nabla \cdot \mathrm{q}\left(\bar{r}, t^{\prime}\right)+u\left(\bar{r}, t^{\prime}\right)\right] d t^{\prime}, \\
\bar{r} \in V, t \geqslant 0 .
\end{gathered}
$$

To complete the mathematical formulation, boundary and initial conditions must be specified. Although any arbitrary boundary conditions may be specified, the advantages of the heat flux formulation become the most apparent for situations involving specified heat fluxes at the boundaries. Equilibrium initial conditions can be readily established as

$$
\begin{aligned}
& \mathbf{q}(\bar{r}, 0)=0, \\
& \frac{\partial \mathbf{q}}{\partial t}(r, 0)=0, \quad \bar{r} \in V .
\end{aligned}
$$

We shall now develop the general solution to a one-dimensional slab problem with the aid of Green's functions. This problem will display the utility and merits of such an alternative formulation in terms of heat flux.

\section{ANALYSIS: ONE-DIMENSIONAL SLAB}

Consider a slab initially at the equilibrium temperature $T_{0}$. At time $t=0$, both external surfaces are suddenly exposed to arbitrary time-dependent heat fluxes while the entire slab is in the presence of a general volumetric heat source. In this situation, the general three dimensional flux formulation developed previously reduces to the following one-dimensional equation for the heat fux distribution

$$
\begin{aligned}
& \frac{\partial^{2} q}{\partial x^{2}}(x, t)-\frac{\partial u}{\partial x}(x, t)=\frac{1}{\alpha}\left(\tau \frac{\partial^{2} q}{\partial t^{2}}(x, t)+\frac{\partial q}{\partial t}(x, t)\right), \\
& x \in(0, l), t>0 .
\end{aligned}
$$

The boundary and initial conditions may be expressed as

$$
\begin{aligned}
& q(0, t)=q_{0}(t), \\
& q(l, t)=q_{l}(t), t>0
\end{aligned}
$$

and

$$
\begin{aligned}
& q(x, 0)=0, \\
& \frac{\partial q}{\partial t}(x, 0)=0, x \in[0, l] .
\end{aligned}
$$

Formally, as $\tau \rightarrow 0$, Eq. (10) reduces to the classical parabolic heat flux formulation. The convenience of the flux formulation for a problem involving specified heat flux boundary conditions now become apparent. That is, a simple boundary condition such as Eq. (11a) replaces a more involved boundary condition

$$
-k \frac{\partial T}{\partial x}(0, t)=\tau \frac{d q_{0}}{d t}(t)+q_{0}(t)
$$

that would be needed when considering the temperature field equation.

For convenience in the subsequent analysis, we introduce the following nondimensional quantities

$$
\eta=c x / 2 \alpha, \quad \eta_{l}=c l / 2 \alpha,
$$

where $c^{2}=\alpha / \tau$ and

$$
\begin{aligned}
& \xi=c^{2} t / 2 \alpha, \\
& \theta(\eta, \xi)=\frac{T(x, t)-T_{0}}{T_{\text {ref }}}, \\
& Q(\eta, \xi)=\frac{q(x, t)}{T_{\text {ref }}(c k / \alpha)},
\end{aligned}
$$

and

$$
S(\eta, \xi)=\frac{u(x, t)}{T_{\mathrm{ref}}\left(k c^{2} / 4 \alpha^{2}\right)},
$$

where $T_{\text {ref }}$ is a reference temperature chosen for convenience.

Introducing the dimensionless quantities, as defined in Eqs. (14a)-(14e) into Eqs. (10)-(12), we obtain the following system of equations governing the dimensionless heat flux distribution:

$$
\begin{aligned}
& \frac{\partial^{2} Q}{\partial \xi^{2}}(\eta, \xi)+2 \frac{\partial Q}{\partial \xi}(\eta, \xi) \\
& \quad=\frac{\partial^{2} Q}{\partial \eta^{2}}(\eta, \xi)-1 / 2 \frac{\partial S}{\partial \eta}(\eta, \xi), \quad \eta \in\left(0, \eta_{l}\right), \quad \xi>0, \\
& Q(0, \xi)=Q_{0}(\xi), \\
& Q\left(\eta_{1}, \xi\right)=Q_{l}(\xi), \quad \xi>0, \\
& \text { and } \\
& \quad Q(\eta, 0)=0, \\
& \quad\left(\frac{\partial Q}{\partial \xi}\right)(\eta, 0)=0, \quad \eta \in\left[0, \eta_{1}\right] .
\end{aligned}
$$

The dimensionless energy balance expressed by Eq. (4) for the one-dimensional case becomes

$$
-\left(\frac{\partial Q}{\partial \eta}\right)(\eta, \xi)+\frac{1}{2} S(\eta, \xi)=\left(\frac{\partial \theta}{\partial \xi}\right)(\eta, \xi),
$$

which can be integrated to give the temperature distribution as 
$\theta(\eta, \xi)=\theta(\eta, 0)-\int_{\xi^{\prime}=0}^{\xi}\left(\frac{\partial Q}{\partial \eta}\left(\eta, \xi^{\prime}\right)+\frac{1}{2} S\left(\eta, \xi^{\prime}\right)\right) d \xi^{\prime}$.

Next we develop the general solution to the system of Eqs. (15)-117) and apply the results for a pulsed surface heat flux.

\section{A. Solution in terms of a Green's function}

The formal starting point in the development of the Green's function method is the application of Green's second formula over the domain of interest ${ }^{32-34}$

$$
\begin{aligned}
\lim _{\epsilon \rightarrow 0} \int_{\xi_{0}=0}^{\xi+\epsilon} \int_{\eta_{0}=0}^{\eta_{t}} G\left(\eta, \xi \mid \eta_{0}, \xi_{0}\right) L_{0}\left[Q\left(\eta_{0}, \xi_{0}\right)\right] d \eta_{0} d \xi_{0} \\
=\lim _{\epsilon \rightarrow 0}\left((B C+I C)+\int_{\xi_{0}=0}^{\xi+\epsilon} \int_{\eta_{0}=0}^{\eta_{t}} Q\left(\eta_{0}, \xi_{0}\right)\right. \\
\left.\quad \times L_{0}^{*}\left[G\left(\eta, \xi \mid \eta_{0}, \xi_{0}\right)\right] d \eta_{0} d \xi_{0}\right) .
\end{aligned}
$$

Here $G\left(\eta, \xi \mid \eta_{0}, \xi_{0}\right)$ is the appropriate Green's function where the arguments are written to represent the "effect/cause" relationship. We introduce $\epsilon>0$ in order to invoke causality at a later time in the analysis.

For any given linear operator $L_{0}$, the contribution of the boundary and initial conditions are represented symbolically by $B C$ and $I C$, respectively. Also, $L_{0}^{*}$ is the formal adjoint operator of the operator $L_{0}$. The operator $L$ is chosen as the modified heat flux linear operator

$$
L \equiv \frac{\partial^{2}}{\partial \eta^{2}}-\frac{\partial^{2}}{\partial \xi^{2}}-2 \frac{\partial}{\partial \xi},
$$

since we are interested in the solution to Eq. (15) which can be expressed as

$$
L[Q(\eta, \xi)]=1 / 2 \frac{\partial S}{\partial \eta}(\eta, \xi), \quad \eta \in\left(0, \eta_{l}\right), \quad \xi>0 .
$$

Integrating the left-hand side of Eq. (20) by parts, and using the linear operator given by Eq. (21) with the understanding that $L_{0}$ represents differentiation with respect to the cause variables $\eta_{0}$ and $\xi_{0}$, we obtain the following explicit expression:

$$
\begin{aligned}
\lim _{\epsilon \rightarrow 0} \int_{\xi_{0}=0}^{\xi+\epsilon} \int_{\eta_{0}=0}^{\eta_{t}} G\left(\eta, \xi \mid \eta_{0}, \xi_{0}\right) L_{0}\left[Q\left(\eta_{0}, \xi_{0}\right)\right] d \eta_{0} d \xi_{0} \\
=\lim _{\epsilon}\left\{\int_{\xi_{0}=0}^{\xi+\epsilon}\left(\left.G \frac{\partial Q}{\partial \eta_{0}}\right|_{0} ^{\eta_{1}}-\left.\frac{\partial G}{\partial \eta_{0}}\right|_{0} ^{\eta_{1}}\right) d \xi_{0}\right. \\
\quad+\int_{\eta_{0}=0}^{\eta_{t}}\left[-G \frac{\partial Q}{\partial \xi_{0}}+Q\left(\frac{\partial G}{\partial \xi_{0}}-2 G\right)\right]_{0}^{\xi+\epsilon} d \eta_{0} \\
\left.\quad+\int_{\xi_{0}=0}^{\xi+\epsilon} \int_{\eta_{0}=0}^{\eta_{t}} Q\left(\eta_{0}, \xi_{0}\right) L *\left[G\left(\eta, \xi \mid \eta_{0}, \xi_{0}\right) d \eta_{0} d \xi_{0}\right]\right\},
\end{aligned}
$$

where the integration shows that the formal adjoint operator of $L_{0}$ is

$$
L_{\mathrm{o}}^{*}=\frac{\partial^{2}}{\partial \eta_{0}^{2}}-\frac{\partial^{2}}{\partial \xi_{0}^{2}}+2 \frac{\partial}{\partial \xi_{0}} .
$$

We observe that $L_{0}^{*} \neq L_{0}$, that is, the operator $L_{0}$ is not formally self-adjoint.

The Green's function is chosen such that

$$
L_{0}^{*}\left[G\left(\eta, \xi \mid \eta_{0}, \xi_{0}\right)\right]=\delta\left(\eta_{0}-\eta\right) \delta\left(\xi_{0}-\xi\right),
$$

subject to homogeneous boundary conditions

$$
G\left(\eta, \xi \mid 0, \xi_{0}\right)=0,
$$

and

$$
G\left(\eta, \xi \mid \eta_{l}, \xi_{0}\right)=0,
$$

with the additional requirement that

$$
G\left(\eta, \xi \mid \eta_{0}, \xi_{0}\right)=0, \quad \xi<\xi_{0},
$$

and

$$
\frac{\partial G}{\partial \xi_{0}}\left(\eta, \xi \mid \eta_{0}, \xi_{0}\right)=0, \quad \xi<\xi_{0}
$$

Conditions (27a) and (27b) represent the causality principle, which is merely a statement that no effect can be experienced prior to a cause. Using the Green's function as governed by Eqs. (25)-(27) and taking the limit as $\epsilon \rightarrow 0$ in Eq. (23), gives the general representation of the heat flux distribution $Q(\eta, \xi)$ as

$$
\begin{aligned}
Q(\eta, \xi)= & 1 / 2 \int_{\xi_{0}=0}^{\xi} \int_{\eta_{0}=0}^{\eta_{1}} G\left(\eta, \xi \mid \eta_{0}, \xi_{0}\right) \frac{\partial S\left(\eta_{0}, \xi_{0}\right)}{\partial \eta_{0}} d \eta_{0} d \xi_{0}+\int_{\xi_{0}=0}^{\xi}\left(\frac{\partial G\left(\eta, \xi \mid \eta_{l}, \xi_{0}\right)}{\partial \eta_{0}} Q\left(\eta_{l}, \xi_{0}\right)-\frac{\partial G\left(\eta, \xi \mid 0, \xi_{0}\right)}{\partial \eta_{0}} Q\left(0, \xi_{0}\right)\right) d \xi_{0} \\
& +\int_{\eta_{0}=0}^{\eta_{l}}\left[-G\left(\eta, \xi \mid \eta_{0}, 0\right) \frac{\partial Q\left(\eta_{0}, 0\right)}{\partial \xi_{0}}+Q\left(\eta_{0}, 0\right)\left(\frac{\partial G\left(\eta, \xi \mid \eta_{0}, 0\right)}{\partial \xi_{0}}-2 G\left(\eta, \xi \mid \eta_{0}, 0\right)\right)\right] d \eta_{0}, \quad \eta \in\left[0, \eta_{l}\right], \quad \xi \geqslant 0 .
\end{aligned}
$$

The only remaining ingredient needed for a complete specification of the function $Q$ is the determination of the Green's function itself, which will be resolved by the finite integral transform technique.

\section{B. Determination of the Green's function}

The finite integral transform technique ${ }^{30}$ is now utilized to determine the Green's function. The appropriate eigenvalue problem obtained from the associated homogeneous version of the system of Eqs. (25), (26), and (27), is given by

$$
\frac{d^{2}}{d \eta_{0}^{2}} \psi_{m}\left(\lambda_{m}, \eta_{0}\right)+\lambda_{m}^{2} \psi_{m}\left(\lambda_{m}, \eta_{0}\right)=0
$$

subject to

$$
\psi_{m}\left(\lambda_{m}, 0\right)=0
$$

and

$$
\psi_{m}\left(\lambda_{m}, \eta_{l}\right)=0 .
$$

The solution is of this problem gives the eigenfunctions

$$
\psi_{m}\left(\lambda_{m}, \eta_{0}\right)=\sin \lambda_{m} \eta_{0},
$$


where the eigenvalues are defined as

$$
\lambda_{m}=\left(m \pi / \eta_{l}\right), \quad m=1,2,3 \ldots
$$

The orthogonality relation is written in terms of the inner product as

$$
\begin{aligned}
& {\left[\psi_{m}\left(\lambda_{m}, \eta_{0}\right), \psi_{n}\left(\lambda_{n}, \eta_{0}\right)\right]} \\
& \equiv \int_{0}^{\eta_{l}} \psi_{m}\left(\lambda_{m}, \eta_{0}\right) \psi_{n}\left(\lambda_{n}, \eta_{0}\right) d \eta_{0} \\
& \quad=\left\{\begin{array}{cc}
N\left(\lambda_{m}\right), & m=n \\
0, & m \neq n
\end{array}\right.
\end{aligned}
$$

where $N\left(\lambda_{m}\right)$ is the normalization integral given by

$$
N\left(\lambda_{m}\right)=\eta_{l} / 2 \text {. }
$$

With the aid of this orthogonality relation, we can now define the finite integral transform pair as follows.

\section{Inversion formula}

$$
G\left(\eta, \xi \mid \eta_{0}, \xi_{0}\right)=\int_{m=1}^{\infty} \frac{\psi_{m}\left(\lambda_{m}, \eta_{0}\right) \bar{G}_{m}\left(\lambda_{m}, \xi_{0}\right)}{N\left(\lambda_{m}\right)}
$$

\section{Integral transform}

$$
\vec{G}_{m}\left(\lambda_{m}, \xi_{0}\right)=\int_{\eta_{0}=0}^{\eta_{l}} \psi_{m}\left(\lambda_{m}, \eta_{0}\right) G\left(\eta, \xi \mid \eta_{0}, \xi_{0}\right) d \eta_{0} .
$$

In order to determine the transform, $\bar{G}_{m}\left(\lambda_{m}, \xi_{0}\right)$, we operate on Eq. (25) with

$$
\int_{\eta_{0}=0}^{\eta_{t}} \psi_{m}\left(\lambda_{m}, \eta_{0}\right) d \eta_{0}
$$

and incorporate the homogeneous boundary conditions of Eqs. (26) and (30) in order to obtain the following ordinary differential equation for $\underline{G}_{m}\left(\lambda_{m}, \xi_{0}\right)$

$$
\begin{aligned}
& \frac{d^{2} \bar{G}_{m}\left(\lambda_{m}, \xi_{0}\right)}{d \xi_{0}^{2}}-2 \frac{d \bar{G}_{m}}{d \xi_{0}}\left(\lambda_{m}, \xi_{0}\right)+\lambda_{m}^{2} \bar{G}_{m}\left(\lambda_{m}, \xi_{0}\right) \\
& =-\delta\left(\xi_{0}-\xi\right) \psi_{m}\left(\lambda_{m}, \eta\right),
\end{aligned}
$$

subject to the transformed initial conditions

$$
\bar{G}_{m}\left(\lambda_{m}, \xi_{0}\right)=0, \quad \xi<\xi_{0},
$$

and

$$
\frac{d \bar{G}_{m}}{d \xi_{0}}\left(\lambda_{m}, \xi_{0}\right)=0, \quad \xi<\xi_{0}
$$

After some careful manipulation, the solution to Eqs. (37) and (38) can be expressed as

$$
\begin{aligned}
\bar{G}_{m}\left(\lambda_{m}, \xi_{0}\right)= & -\frac{\psi_{m}\left(\lambda_{m}, \eta\right) e^{-\left(\xi-\xi_{0}\right)}}{\sqrt{\lambda_{m}^{2}-1}} \\
& \times \sin \sqrt{\lambda_{m}^{2}-1}\left(\xi-\xi_{0}\right), \quad \xi>\xi_{0} .
\end{aligned}
$$

Substituting Eq. (39) into the inversion formula [Eq. (34)], we obtain the Green's function as

$$
G\left(\eta, \xi \mid \eta_{0}, \xi_{0}\right)=-\sum_{m=1}^{\infty} \frac{\psi_{m}\left(\lambda_{m}, \eta\right) \psi_{m}\left(\lambda_{m}, \eta_{0}\right) e^{-\left(\xi-\xi_{0}\right)} \sin \sqrt{\lambda_{m}^{2}-1}\left(\xi-\xi_{0}\right)}{N\left(\lambda_{m}\right) \sqrt{\lambda_{m}^{2}-1}}, \quad \xi>\xi_{0}, \quad \eta \in\left[0, \eta_{l}\right],
$$

where the eigenfunctions $\psi_{m}\left(\lambda_{m}, \eta\right), \quad \psi_{m}\left(\lambda_{m}, \eta_{0}\right)$ are defined by Eq. (31), the eigenvalues are expressed by Eq. (32).

Since the Green's function is now known, the heat flux distribution $Q(\eta, \xi)$ for any arbitrary volumetric heat source and boundary conditions as expressed as Eq. (16) can be obtained from Eq. (28). The dimensionless temperature distribution can be determined by using Eq. (19). A specific example is investigated to display the utility of this heat flux formulation to bring forth some unique features of heat conduction based on the hyperbolic formulation.

\section{Puised surface heat flux}

Here we consider a flat plate of thickness $l$, subject to a pulsed heat flux at the surface $x=0$ which has an intensity $q_{0}$ for a duration of $\Delta t$ seconds. The surface at $x=l$ is insulated for all time $t>0$. Since a linear model is considered, the effect of a thermal disturbance at $x=l$ would merely be superimposed with the effect due to the disturbance at $x=0$. The body is initially in equilibrium at the uniform temperature $T_{0}$ and contains no volumetric heat source. Previous investigation ${ }^{20-22}$ of the hyperbolic surface heat flux problem, based on the temperature formulation, have not considered the heat flux distribution in any manner. Boundary conditions involving heat flux have been incorrectly formulated ${ }^{22}$ by using Fourier's law at the boundaries in conjunction with the hyperbolic model inside the region, re- sulting in an inconsistent formulation leading to questionable results.

For this specific case, a convenient reference temperature is chosen as

$$
T_{\mathrm{ref}}=\frac{q_{0}}{c k / \alpha} .
$$

The dimensionless heat flux field equation [Eq. (15)] reduces to

$\frac{\partial^{2} Q}{\partial \eta^{2}}(\eta, \xi)=\frac{\partial^{2} Q}{\partial \xi^{2}}(\eta, \xi)+2 \frac{\partial Q}{\partial \xi}(\eta, \xi), \quad \eta \in\left(0, \eta_{l}\right), \quad \xi>0 .(42)$

The boundary conditions are expressed as

$$
\begin{aligned}
& q_{0}(0, t)=q_{0}[H(t)-H(t-\Delta t)], \\
& q_{0}(l, t)=0, \quad t>0,
\end{aligned}
$$

or in dimensionless form as

$$
Q(0, \xi)=H(\xi)-H(\xi-\Delta \xi),
$$

and

$$
Q\left(\eta_{l}, \xi\right)=0, \quad \xi>0
$$

where $H$ represents the Heavyside step function. It is interesting to note that the proper boundary condition, corresponding to Eq. (43a), in the temperature formulation would be

$$
\frac{\partial T}{\partial x}(0, t)=-\frac{q_{0}}{k}[H(t)-H(t-\Delta t)
$$




$$
+\tau \delta(t)-\tau \delta(t-\Delta t)]
$$

The solution to the pulsed heat flux problem is now obtained from the general formula solution Eq. (28), by using the Green's function, Eq. (40), the boundary and initial con- ditions given by Eqs. (44) and (17), respectively, and a zero source. Performing the indicated operations and after a lengthy but straightforward set of manipulations, the heat flux distribution becomes

$$
\begin{aligned}
Q(\eta, \xi)= & \left(1-\frac{\eta}{\eta_{l}}\right)[H(\xi)-H(\xi-\Delta \xi)]-\frac{2}{\eta_{l}} \sum_{m=1}^{\infty} \frac{\sin \lambda_{m} \eta}{\lambda_{m}} e^{-\xi}\left(\frac{\sin \sqrt{\lambda_{m}^{2}-1} \xi}{\sqrt{\lambda_{m}^{2}-1}}+\cos \sqrt{\lambda_{m}^{2}-1} \xi\right) H(\xi) \\
& +\frac{2}{\eta_{l}} \sum_{m=1}^{\infty} \frac{\sin \lambda_{m} \eta}{\lambda_{m}} e^{-(\xi-\Delta \xi)}\left(\frac{\sin \sqrt{\lambda_{m}^{2}-1}(\xi-\Delta \xi)}{\sqrt{\lambda_{m}^{2}-1}}+\cos \sqrt{\lambda_{m}^{2}-1}(\xi-\Delta \xi)\right) H(\xi-\Delta \xi), \quad \eta \in\left[0, \eta_{l}\right], \quad \xi \geqslant 0
\end{aligned}
$$

The dimensionless temperature distribution is now obtained by substituting Eq. (46) into Eq. (19), with no source term, and again performing the indicated operations to arrive at ${ }^{35}$

$$
\begin{aligned}
\theta(\eta, \xi)= & \left(\frac{\xi}{\eta_{l}}+\eta^{2}-2 \eta+\frac{2 \eta_{l}}{3}\right) H(\xi)-H(\xi-\Delta \xi)-\frac{4}{\eta_{l}} \sum_{m=1}^{\infty} \frac{e^{-\xi} \cos \lambda_{m} \eta}{\lambda_{m}^{2}}\left(\frac{\left(2-\lambda_{m}^{2}\right)}{2 \sqrt{\lambda_{m}^{2}-1}} \sin \sqrt{\lambda_{m}^{2}-1 \xi}\right. \\
& \left.+\cos \sqrt{\lambda_{m}^{2}-1} \xi\right) H(\xi)+\frac{\Delta \xi}{\eta_{l}} H(\xi-\Delta \xi)+\frac{4}{\eta_{l}} \sum_{m=1}^{\infty} \frac{e^{-(\xi-\Delta \xi)} \cos \lambda_{m} \eta}{\lambda_{m}^{2}} \\
& \times\left(\cos \sqrt{\lambda_{m}^{2}-1}(\xi-\Delta \xi)+\frac{\left(2-\lambda_{m}^{2}\right)}{2 \sqrt{\lambda_{m}^{2}-1}} \sin \sqrt{\lambda_{m}^{2}-1}(\xi-\Delta \xi)\right) H(\xi-\Delta \xi), \quad \eta \in\left[0, \eta_{l}\right], \quad \xi \geqslant 0 .
\end{aligned}
$$

The heat flux and temperature distributions, as predicted by the hyperbolic and parabolic heat conduction equations, are now numerically examined for the surface pulsed. heat flux problem.

\section{RESULTS}

Numerical results displaying the development of the heat flux and temperature distributions arising from a pulsed surface heat flux of duration $\Delta \xi=0.2$ on a slab of thickness $\eta_{l}=1$ are now presented. The hyperbolic and parabolic solutions are then compared showing the distinct differences in the two heat conduction approximations.

An interesting comparison can be made between linear parabolic and hyperbolic heat conduction concerning the rate of convergence of their respective infinite series solutions. In parabolic heat conduction, the bilinear series solutions converge very rapidly. This rapid convergence rate is attributed to the decaying exponential term which contains the eigenvalues. However, in hyperbolic heat conduction no such term exists as demonstrated in Eqs. (46) and (47) displaying the heat flux and temperature, respectively. These bilinear forms require hundreds of terms to obtain three significant figures of accuracy. However, techniques such as the Kummer transform may be incorporated to accelerate the rate of convergence of these series solutions.

Figure 1 displays the heat flux distribution for both the hyperbolic and parabolic cases at various times $\xi$. The hyperbolic solution shows that for $\xi<\eta_{1}=1$, an undisturbed region exists ahead of the wave front. Since no molecular communication has occurred ahead of the front, the behavior of the heat flux is the same as would exist in the half-space problem. Certainly a finite speed of propagation is now associated with the rate of heat flow in the medium. As the wave propagates forward, energy is deposited in the wake by diffusion. The absolute magnitude of the wave front decreases exponentially with increasing time due to the dissipation of energy by diffusion. On the other hand, the parabolic solution predicts that heat will propagate with an infinite speed and will be felt instantaneously throughout the medium after a thermal disturbance has been introduced.

When the wave front impacts the insulated surface at $\xi=\eta_{l}=1$, the energy will refiect back toward the origin at

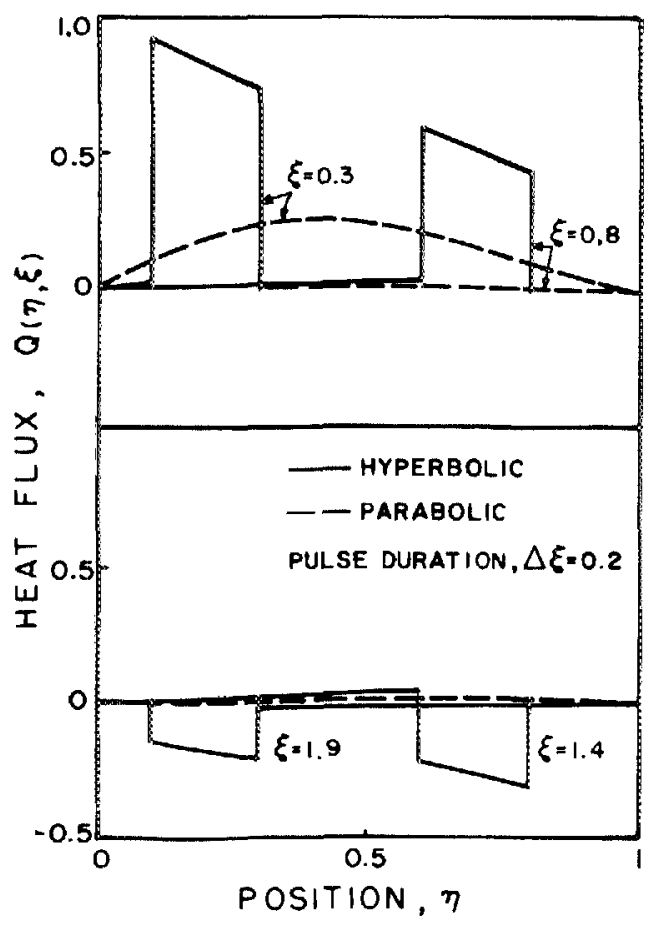

FIG. 1. Heat flux distribution resulting from a pulsed surface heat flux in a slab of thickness $\eta_{t}=1$. 


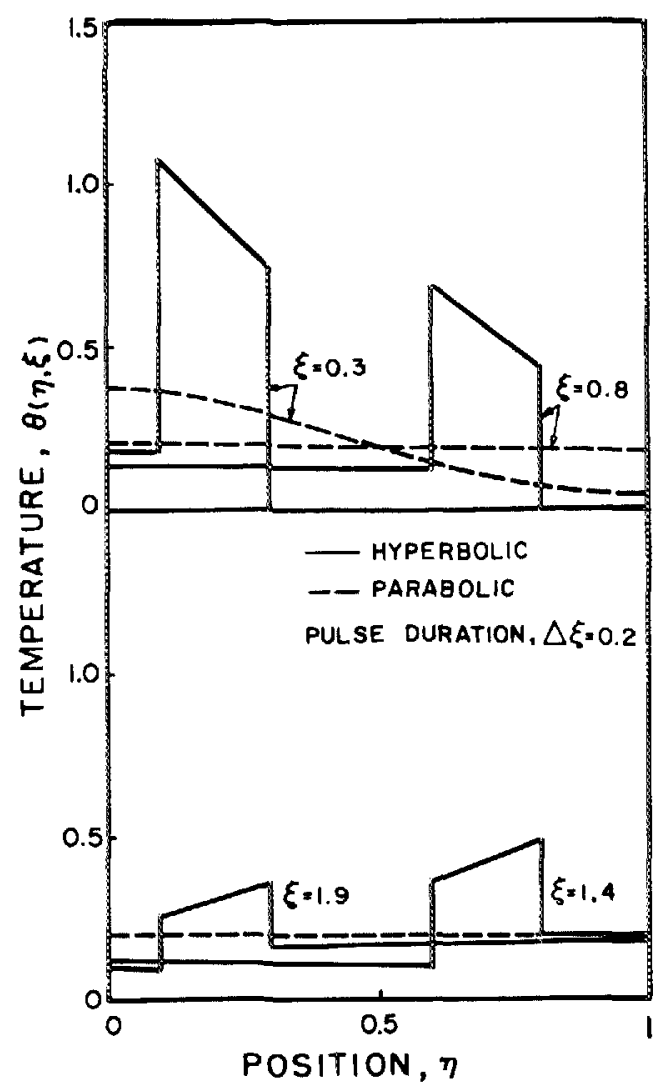

FIG. 2. Temperature distribution resulting from a pulsed surface heat flux in a slab of thickness $\eta_{l}=1$.

$\eta=0$. This process of reflections at the surfaces will persist until the diffusion phenomena dominates. In contrast, the parabolic heat flux distribution displays monotonic decay to its steady-state value, i.e., it only predicts heat flow from $\eta=0$ to $\eta=1$ for all time $\xi$.

The corresponding temperature distributions $(\Delta \xi=0.2)$ are shown in Fig. 2. Again, the distinct wave na. ture associated with hyperbolic heat conduction dominates Diffusion causes the tersperature wave to exponentially decay with time in the direction of propagation. Since energy has been deposited behind the wave front, a small residual temperature is present. The parabolic solution displays a rapid decay to its steady-state value of $\Delta \xi / \eta_{t}$. These two figures distinctly show the dominant wave feature associated to the hyperbolic heat conduction approximation.

\section{COMCLUSIONS}

A general three-dimensional constant property heat flux formulation based on the hyperbolic heat conduction approximation has been developed. This formulation is a viable alternative to the classical temperature formulation in many incidences. The equivalent heat flux formulation for the parabolic heat conduction approximation can be obtained by letting the relaxation time $\tau$ tend to zero. In situations involving specified heat flux boundary condition, this formulation is especially appealing as demonstrated in the one-dimensional slab problem. It has been shown that the presence of a volumetric heat source in a medium will appear as the gradient of the source in the governing flux vector equation, while the source and its time derivative shall be realized in the corresponding temperature field equation.
The temperature distribution is obtained by integrating the energy conservation law over time.

The Green's function method, as developed from Green's second formula, has been applied to determine the heat flux distribution in a finite slab. A general expression for the heat flux distribution subject to any volumetric energy source and boundary conditions of the first kind (in flux) has been established. The determination of the Green's function has been obtained by the finite integral transform technique in the cause variable without introducing the standard reciprocity relation. Certainly, the development of the Green's function in this manner is more natural from the mathematical point of view.

The wave feature of the hyperbolic heat conduction approximation has been demonstrated in the finite thickness slab subject to a pulsed surface heat flux. The realization of a finite speed of propagation of the thermal waves has been confirmed through this example. This, of course, is in contrast to the linear parabolic approximation which predicts an infinite speed of heat propagation.

\section{ACKNOWLEDGMENT}

This work was supported through the National Science Foundation Grant NSF MEA 83-13301.

'S. Simons, Trans. Theory Stat. Phys. 2, 117 (1972).

${ }^{2}$ M. Chester, Phys. Rev. 131, 2013 (1963).

${ }^{3}$ Yehuda Taitel, Int. J. Heat Mass Transfer 15, 369 (1972).

${ }^{4}$ J. C. Maxwell, Philos. Trans. R. Soc. London 157, 49 (1867).

${ }^{5}$ M. P. Vernotte, C. R. 247, 2103 (1958).

${ }^{6} M$. P. Vernotte, C. R. 246, 3154 (1958).

${ }^{7}$ M. P. Vernotte, C. R. 252, $2190(1961)$

${ }^{8}$ Cattaneo, C. R. 247, 431 (1958).

${ }^{9} \mathrm{P}$. M. Morse and H. Fesbach, Methods of Theoretical Physics, $I$ (McGrawHill, New York, 1953).

${ }^{10}$ H. D. Weyman, Am. J. Phys. 36, 488 (1967).

${ }^{11}$ S. Kaliski, Bull. Acad. Pol. Sci. Ser Sci. Tech. 13, 211 (1965).

${ }^{12}$ M. E. Gurtin and A. C. Pipkin, Arch. Rational Mech. Anal. 31, 113 (1968).

${ }^{13}$ V. A. Bubnov, Int. J. Heat Mass Transfer 19, 175 (1976).

${ }^{14}$ B. M. Berkovsky and V. G. Bashtovoi, Int. J. Heat Mass Transfer 20, 621 (1977).

${ }^{13}$ N. G. Van Kampen, Physica 46, 315 (1970)

${ }^{16}$ D. C. Kelly, Am. J. Phys. 36, 585 (1968).

${ }^{17}$ B. Vick and M. N. Özisik, J. Heat Transfer 105, 902 (1983).

${ }^{18}$ M. N. Özisik and B. Vick, Int. J. Heat Mass Transfer 27, 1845 (1984).

${ }^{19} \mathrm{E}$. Lorenzini and M. Spiga, Warme. Stoffubertrag. 16, 113 (1982).

${ }^{20} \mathrm{R}$. Domanski, 6th International Heat Transfer Conference, Toronto Ontario (National Resource Council of Canada, Toronto, Ontario, 1978), p. 275.

${ }^{21}$ M. J. Maurer and H. A. Thompson, J. Heat Transfer 95, 284 (1973).

${ }^{22}$ J. P. Brazel and E. J. Nolan, 6th Conf. On Thermal Conductivity (1966), p. 238.

${ }^{23}$ K. J. Baumeister and T. D. Hamill, J. Heat Transfer 91, 543 (1969).

${ }^{24}$ M. S. Kazimi and C. A. Erdman, J. Heat Transfer 97, 615 (1975).

${ }^{25}$ S. H. Chan, J. D. Low, and W. K. Mueller, AIChE 17, 1499 (1971).

${ }^{26}$ Tsai-tse Kao, J. Heat Transfer 99, 343 (1977).

${ }^{27}$ K. J. Baumeister and T. D. Hamill, J. of Heat Transfer 93, 126 (1971).

${ }^{28}$ D. C. Wiggert, J. Heat Transfer 99, 35 (1977).

${ }^{29}$ G. F. Carey and M. Tsai, Numerical Heat Transfer 5, 309 (1982).

${ }^{30}$ M. N. Özisik, Heat Conduction (Wiley, New York, 1980).

${ }^{31}$ V. Arpaci, Conduction Heat Transfer (Addison-Wesley, California, 1966).

${ }^{32}$ M. D. Greenberg, Application of Green's Functions in Science and Engineering (Prentice-Hall, New Jersey, 1971).

${ }^{33}$ G. F. Roach, Green's Functions, 2nd ed. (Cambridge University, London, 1982).

${ }^{34}$ F. Hildebrand, Advanced Calculus for Applications, 2nd ed. (PrenticeHall, New Jersey, 1976).

${ }^{35}$ L. B. W. Jolley, Summation of Series (Dover, New York, 1961), p. 62. 